Bio - grafia. Escritos sobre la Biología y su Enseñanza. ISSN 2027

Edición Extraordinaria. p.p. $636-650$

Memorias del VIII Encuentro Nacional de Experiencias en Enseñanza de la Biología y la Educación Ambiental. III Congreso Nacional de Investigación en Enseñanza de la Biología.

\title{
EDUCACIÓN AMBIENTAL PARA LA CONSERVACIÓN DE LA FAUNA VERTEBRADA EN NORCASIA-SAMANÁ (CALDAS)
}

\section{ENVIRONMENTAL EDUCATION TO CONSERVATION OF WILDLIFE VERTEBRATE IN NORCASIA-SAMANA (CALDAS)}

\author{
Estefanía Espitia Martínez; : Leidis Patricia Toro Julio²; Laura Aponte Moreno33; Beatriz \\ Toro Restrepo 4 \\ ${ }^{1}$ estephannie-espitia@hotmail.com ${ }^{2}$ leidis0826@hotmail.com ${ }^{3}$ laura.aponte@ucaldas.edu.co \\ 할eatriz.toro@ucaldas.edu.co
}

\section{RESUMEN}

La educación ambiental ha ido cambiando desde un enfoque conservacionista a hacia una actitud crítica, una capacidad creadora y un nuevo sistema de valores y de comportamientos en los sujetos, lo que es esencial para una conservación integral. Por lo tanto, el objetivo del estudio fue sensibilizar a las comunidades sobre la importancia de conocer, proteger y conservar la fauna de la región. El área de estudio se encuentra ubicada en el departamento de Caldas en los municipios de Samaná y Norcasia, específicamente en el trasvase de las aguas del río Manso al embalse Amaní que sirve a la Central Hidroeléctrica Miel I de la empresa ISAGEN. El trabajo se realizó en 15 veredas, en las que se hizo un diagnóstico participativo complementado con una encuesta estructurada, analizando las variables univariadas y bivariadas en el programa SPSS. Tanto con la población adulta como infantil se realizaron actividades de conservación, integración y sensibilización ambiental, las cuales fueron analizadas mediante la Matriz de Marco Lógico. 
Bio - grafia. Escritos sobre la Biología y su Enseñanza. ISSN 2027

Edición Extraordinaria. p.p. 636 - 650

Memorias del VIII Encuentro Nacional de Experiencias en Enseñanza de la Biología y la Educación Ambiental. III Congreso Nacional de Investigación en Enseñanza de la Biología.

Se encontró que en la zona las principales causas de la pérdida de biodiversidad son la tala, la caza, las quemas y la ganadería. No obstante, la comunidad tiene buen conocimiento sobre la fauna silvestre lo que ha facilitado una mayor valoración y mejor protección de la misma. Con el trabajo realizado, no sólo se ha contribuido a un mayor conocimiento de la fauna local, una mayor sensibilidad de la comunidad, sino la formación de líderes ambientales que harán sostenible y continuo el proceso de educación ambiental.

Palabras clave: Educación ambiental, fauna silvestre, conservación, trasvase río Manso, ISAGEN.

\begin{abstract}
Environmental education has been changing from a conservation perspective towards a critical and creative attitude. There is a new system of values and behaviors in people, which contribute to a holistic conservation. Therefore, the goal was to sensitize people on the importance of knowing, protect and conserve wildlife in the region. The area study it is located in the department of Caldas on the municipalities of Samana and Norcasia, specifically, in the zone of Manso River diverts to the Amani reservoir that serves the Miel I Hydroelectric Power Plant of ISAGEN Company. The work was conducted in 15 villages, where it made a participatory diagnosis was complemented with a structured survey, analyzing univariate and bivariate variables in SPSS program. With adults and child was made conservation activities, integration, and environmental awareness. These were analyzed using the Logical Framework Approach. It was found that in the major causes of biodiversity loss are logging, hunting, burning and livestock. However, the community has good knowledge about wildlife which it has facilitated a major valuation and better protection of it. The work has not only contributed to a better understanding of the local fauna and major sensitivity of the community.
\end{abstract}


Bio - grafia. Escritos sobre la Biología y su Enseñanza. ISSN 2027

Edición Extraordinaria. p.p. 636 - 650

Memorias del VIII Encuentro Nacional de Experiencias en Enseñanza de la Biología y la Educación Ambiental. III Congreso Nacional de Investigación en Enseñanza de la Biología.

It is also training environmental leaders, which will facilitate a continuous and sustainable process of environmental education.

Key words: environmental education, wildlife conservation, Manso River diverts, ISAGEN.

\section{INTRODUCCIÓN}

La educación ambiental (EA) considera el ambiente como un todo involucrando a la población en general y representa una realidad compleja que debe ser explicada y completada por la integración de saberes teóricos y prácticos dando solución a problemas ambientales concretos. En ella se generan conocimientos, valores, actitudes y habilidades que contribuyen en la toma de decisiones y la participación activa y organizada (Terrón, 2000; Martínez, 2005 \& García, 2003). En los últimos años ha surgido un intenso debate y crítica a nivel nacional e internacional sobre la naturaleza de la educación ambiental, considerando que en la próxima década ésta debe reorientarse hacia mejorar la calidad de vida de todos los ciudadanos (Tilburry, 1995). El impacto que ha tenido la EA en las comunidades ha sido positivo ya que es considerada como una prioridad para aminorar los impactos ambientales (Castelltort et al., 2005).

Éste es el caso del oriente de Caldas, cuya principal actividad económica es eminentemente agropecuaria con predominio de áreas en pastos que ocupan la mayor superficie. La ampliación de la frontera agrícola y la continua degradación del territorio han ocasionado una disminución en la biodiversidad (Alzate, 2001). Así mismo, la urbanización, las infraestructuras, la caza, la deforestación, las quemas y la falta de voluntad de las personas ponen en una situación crítica la permanencia de las especies y de los ecosistemas que las sustentan (Fundación IPADE, 2010). Además, en la zona se han registrado especies 
Bio - grafia. Escritos sobre la Biología y su Enseñanza. ISSN 2027

Edición Extraordinaria. p.p. 636 - 650

Memorias del VIII Encuentro Nacional de Experiencias en Enseñanza de la Biología y la Educación Ambiental. III Congreso Nacional de Investigación en Enseñanza de la Biología.

migratorias, endémicas, en estado de amenaza y vulnerabilidad y nuevos registros (ISAGEN, 2012).

Según lo anterior, la EA se convierte en una herramienta para aminorar los impactos existentes sobre la fauna convirtiéndose en un proceso educativo integral $e$ interdisciplinario. Ésta juega un papel decisivo para alcanzar los objetivos de la conservación, protección del medio ambiente y el desarrollo sostenible, ya que busca mejorar el manejo de los recursos naturales y reducir los daños al ambiente y como estos perjudican también al ser humano (Martínez, 2005; Rojas, Isola, Gómez \& Águila, 2007). Por lo tanto, y considerando que desde el 2011 en las comunidades aledañas a la Central Hidroeléctrica Miel I y los trasvases Guarinó y Manso, los trabajos en educación ambiental han sido enfocados al fortalecimiento de los proyectos ambientales escolares (PRAE) y al establecimiento de huertas comunitarias (UCO, 2013a), el presente proyecto buscó sensibilizar a las comunidades sobre la importancia de conocer, proteger y conservar la fauna silvestre en las zonas donde tiene influencia directa el transvase Manso con el fin de articular los procesos y actores, contribuyendo con la conservación ambiental.

\section{METODOLOGÍA}

Área de estudio: El trasvase del río Manso se encuentra localizado en el departamento de Caldas, en límites de los municipios de Samaná y Norcasia, en la vertiente oriental de la Cordillera Central (Figura 1). La zona del trasvase está centrada en el corregimiento de Berlín, localizado a $15 \mathrm{~km}$ del municipio de Norcasia. La zona de vida en el área corresponde al bosque húmedo tropical (bh-T) (Holdridge, 1982). Se trabajó en 15 veredas, seis aguas arriba del trasvase del río Manso (La Reforma, La Argentina, La Sonrisa, Berlín, Piedras Verdes y San Diego) y nueve veredas aguas abajo (Las Delicias, Manizalitos, Jagual Alto y Bajo, Quiebra de Roque, La Samaria, La Estrella, Cadenales y Risaralda). 
Bio - grafia. Escritos sobre la Biología y su Enseñanza. ISSN 2027

Edición Extraordinaria. p.p. 636 - 650

Memorias del VIII Encuentro Nacional de Experiencias en Enseñanza de la Biología y la Educación Ambiental. III Congreso Nacional de Investigación en Enseñanza de la Biología.

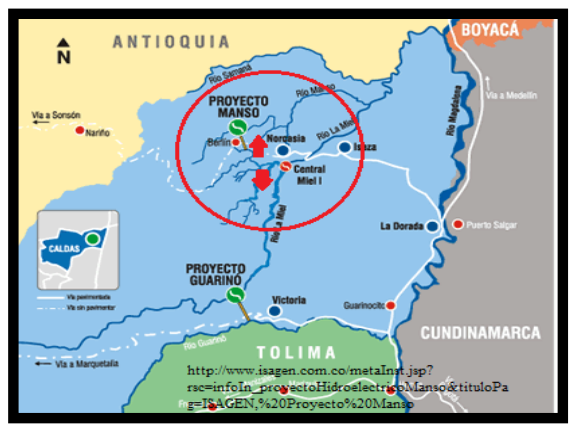

Figura 1. Área de estudio municipios Samaná y Norcasia. Tomado de ISAGEN 2014.

\section{Estrategia educativa}

El trabajo fue realizado durante un año y comprendió una fase diagnóstica y una de sensibilización comunitaria. Se conformaron grupos de mínimo cinco personas. El desarrollo de las actividades se hizo tanto con población infantil como adulta y los grupos de trabajo se establecieron como "grupos ecológicos".

Se desarrollaron seis actividades: 1) Socialización del proyecto; 2) Diagnóstico participativo mediante la metodología de árbol de problemas completándose con una encuesta estructurada; 3) Conociendo para conservar donde se resaltó la importancia de las especies en el ecosistema; 4) Expedición ambiental, cuyo objetivo reconocer en campo las problemáticas de la zona y los servicios; y 5) Mi mundo en imágenes, con la cual se hizo una reconstrucción de la memoria a través de las fotografías tomadas; y 6) Conversatorio ambiental. Estas actividades fueron complementadas con la integración de las comunidades con investigadores que trabajan en la zona en pro de la conservación a través de un foro 
Bio - grafia. Escritos sobre la Biología y su Enseñanza. ISSN 2027

Edición Extraordinaria. p.p. 636 - 650

Memorias del VIII Encuentro Nacional de Experiencias en Enseñanza de la Biología y la Educación Ambiental. III Congreso Nacional de Investigación en Enseñanza de la Biología.

ambiental y un festival ambiental del Tití gris donde se trabajó en articulación con algunas entidades de carácter público y privado que trabajan en la zona.

\section{Análisis de los datos}

La encuesta estructurada constó de 17 preguntas abiertas y cerradas para evaluar el conocimiento de las personas con respecto a la fauna y a las principales problemáticas ambientales. Para su análisis se utilizó el programa SPSS versión 18 (PAWS Stactistics 18). Además, para el análisis de las actividades enfocadas hacia la conservación se hizo mediante la Matriz de Marco Lógico.

\section{RESULTADOS Y DISCUSIÓN}

Socialización: La actividad de socialización logró un acercamiento con las personas de las comunidades con el fin de establecer buenas relaciones, crear compromisos y responsabilidades en las personas frente al medio ambiente. La comunicación fue muy importante en esta actividad ya que permitió crear una conexión entre la comunidad y el personal de la Universidad de Caldas, lo que permitió un mejor establecimiento del programa de educación ambiental en la zona. Así lo confirma Berrenechea, (2014), quien dice que la comunicación es un proceso dinámico en el sentido que genera cambios en la interacción, en el otro y en nosotros mismos. Además, las comunidades que trabajan en equipo indican que asumen responsabilidades y trabajan voluntariamente en el tiempo previsto (Cándelo, Ortiz \& Unger, 2003). 
Bio - grafia. Escritos sobre la Biología y su Enseñanza. ISSN 2027

Edición Extraordinaria. p.p. 636 - 650

Memorias del VIII Encuentro Nacional de Experiencias en Enseñanza de la Biología y la Educación Ambiental. III Congreso Nacional de Investigación en Enseñanza de la Biología.

\section{Fase diagnóstica}

Diagnóstico participativo: pese a que las comunidades ya tienen conocimiento de las problemáticas que enfrentan, el diagnóstico mediante el árbol de problemas permitió identificar más claramente las causas y consecuencias directas de la pérdida biodiversidad. Se identificaron 21 causas, siendo las principales el desconocimiento, la falta de conciencia ambiental, la poca voluntad, el desempleo, la agricultura, la ganadería, el tráfico de fauna, la caza, entre otras (Figura 2). En cuanto a los efectos se reconocieron 14 consecuencias, siendo las principales la pérdida de fauna y flora identificada en el $100 \%$ de las veredas seguida de la erosión del suelo $(87 \%)$ y de la pérdida de nutrientes del suelo y el aumento de calor (80\%). Las personas de las comunidades manifestaron que el entorno ha tenido muchos cambios en el tiempo y consideraron como una evidencia de esto el hecho de que anteriormente se presentaban en la zona unas mejores cosechas, un clima más agradable y una mayor presencia de bosques y de animales.

El análisis de la encuesta arrojó que a medida que aumenta el nivel de escolaridad y la permanencia de las personas en la zona hace que recuerdan más los programas de conservación ejecutados por las entidades que han trabajado en la zona. En cuanto al conocimiento de la fauna, se encontró que Dinomys branickii (Guagua), Dasyprocta fuliginosa (Guatín) y Dasypus novemcinctus (Armadillo) son las especies que han presentado disminución de sus poblaciones en el área de influencia del trasvase Manso, ya que los habitantes de las veredas reconocen haber dejado de ver individuos de la especie.

\section{Mi Mundo en Imágenes}

Población adulta. En las 15 veredas fueron tomadas 1045 fotografías, de las cuales el $75 \%$ fueron relacionadas con la biodiversidad, el $15 \%$ representó su cotidianidad con fotos en su mayoría de fauna doméstica y el $10 \%$ fotografió las problemáticas de la zona como la 
Bio - grafia. Escritos sobre la Biología y su Enseñanza. ISSN 2027

Edición Extraordinaria. p.p. 636 - 650

Memorias del VIII Encuentro Nacional de Experiencias en Enseñanza de la Biología y la Educación Ambiental. III Congreso Nacional de Investigación en Enseñanza de la Biología.

quema, la tala, la disminución del agua y la ganadería. Se encontró que las percepciones que tienen las personas de la zona de influencia en su mayoría se relacionan con la biodiversidad (75\%), lo que indica que su entorno es de gran importancia para su bienestar y demuestran el un arraigo que tienen con su entorno.

Población infantil. En el primer momento de la actividad "Mi animal favorito" se identificaron 39 especies de fauna silvestre representados en tres de los cuatro grupos con que se viene trabajando en el programa, siendo los mamíferos los más representativos con 22 spp, seguido de las aves con 12 spp y los reptiles con cinco. Los niños no identifican a ninguna especie del grupo de los anfibios como su animal favorito. De la actividad "Dibujando mi paisaje" se obtuvieron 188 dibujos, de los cuales 168 se relacionaron con el ambiente y 35 incluían fauna silvestre y tres de fauna exótica, lo que indica que los niños reconocen más la fauna de la región por estar en una interacción directa con ésta.

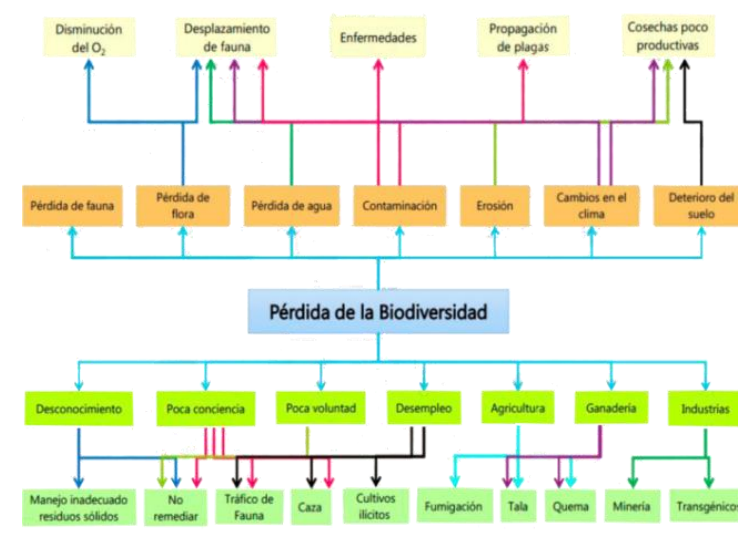

Figura 2. Árbol de problemas participativo en relación a la Pérdida de biodiversidad, realizado en veredas del área de influencia del Trasvase del Río Manso. 
Bio - grafia. Escritos sobre la Biología y su Enseñanza. ISSN 2027

Edición Extraordinaria. p.p. 636 - 650

Memorias del VIII Encuentro Nacional de Experiencias en Enseñanza de la Biología y la Educación Ambiental. III Congreso Nacional de Investigación en Enseñanza de la Biología.

\section{Fase de Conservación}

Conociendo para conservar: Se hizo un ejercicio de reconstrucción de la memoria colectiva de la fauna silvestre local obteniendo de manera participativa un total de 73 especies de aves, 41 especies de herpetos y 23 especies de mamíferos, al mismo tiempo se logró identificar los servicios ambientales prestados por cada uno de estos grupos en los que se destacaron la dispersión de semillas por parte de las aves y de algunos mamíferos, la polinización y el control de plagas. En esta actividad la ayuda de medios audiovisuales permitió a las personas de las comunidades un entendimiento sobre los cuatros grupos trabajados, los servicios ambientales que prestan en el ambiente y a su vez reflexionaron sobre el tema de contaminación ambiental. Según Tobasura, (2004) la enseñanza audiovisual conforma un cuerpo del saber transmisible con contenidos, estrategias y metodologías propias.

Expedición ambiental. La actividad buscó confrontar la información que se había dado en las diferentes actividades con la realidad de su entorno (Figura 3). Se hizo un ejercicio de "reconstrucción colectiva del paisaje" con el cual se logró hacer una comparación del cambio del entorno a través del tiempo, donde las personas manifestaron que antes había más vegetación, animales y agua y reflexionaron que la principal causa de tal cambio es el hombre. También, se trabajó "Problemáticas de mi vereda" en el que las comunidades despejaron dudas acerca de las problemáticas ambientales tales como la disminución del recurso hídrico, la tala del bosque, caza, tráfico y tenencia ilegal de especies. Según estudios realizados por López, (2000) las salidas de campo rompen la rutina habitual y trasladan el aprendizaje y el conocimiento al mundo real. 
Edición Extraordinaria. p.p. 636 - 650

Memorias del VIII Encuentro Nacional de Experiencias en Enseñanza de la Biología y la Educación Ambiental. III Congreso Nacional de Investigación en Enseñanza de la Biología.

Con el fin de conocer si todas las actividades realizadas habían llevado a un cambio en las personas, nuevamente se hizo un pequeño cuestionario en el cual se identificaron varias problemáticas que fueron comparadas con las encontradas en la realización de la encuesta en la actividad de "diagnóstico participativo". Se encontró que al principio el mayor problema eran las basuras (43\%), seguido de la deforestación (35\%) y las quemas (25\%), pero en el cuestionario la problemática principal identificada fue la deforestación con un $76 \%$, seguido de las quemas (55\%). Lo anterior lleva a pensar que a medida que se fueron realizando las actividades de sensibilización con las comunidades, estas fueron concientizándose de otras problemáticas que afectan la fauna silvestre local y que para el oriente de Caldas son más evidentes (Figura 4).
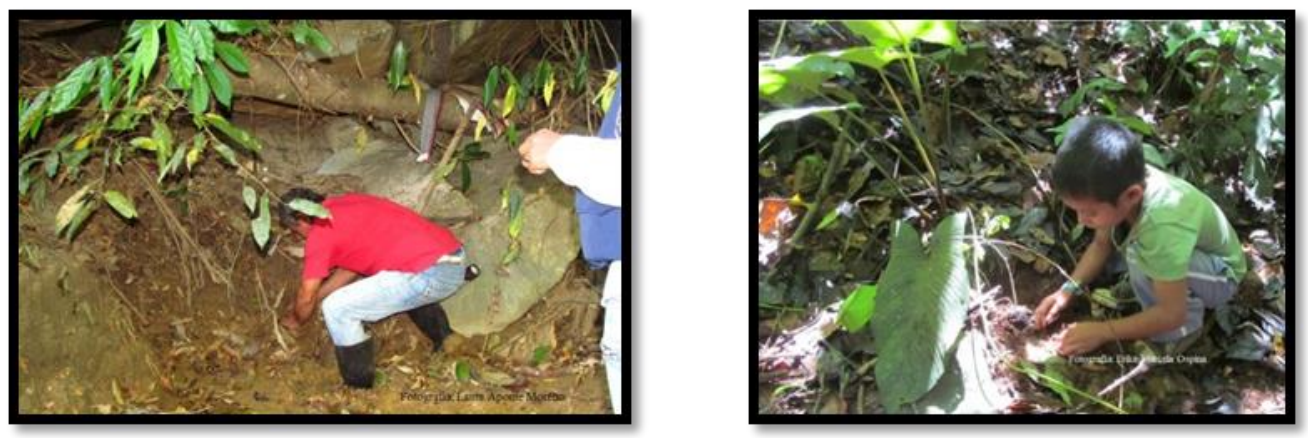

Figura 3. Siembra de árboles por parte de las comunidades de la vereda Manizalitos y La Reforma. 
Bio - grafia. Escritos sobre la Biología y su Enseñanza. ISSN 2027

Edición Extraordinaria. p.p. 636 - 650

Memorias del VIII Encuentro Nacional de Experiencias en Enseñanza de la Biología y la Educación Ambiental. III Congreso Nacional de Investigación en Enseñanza de la Biología.

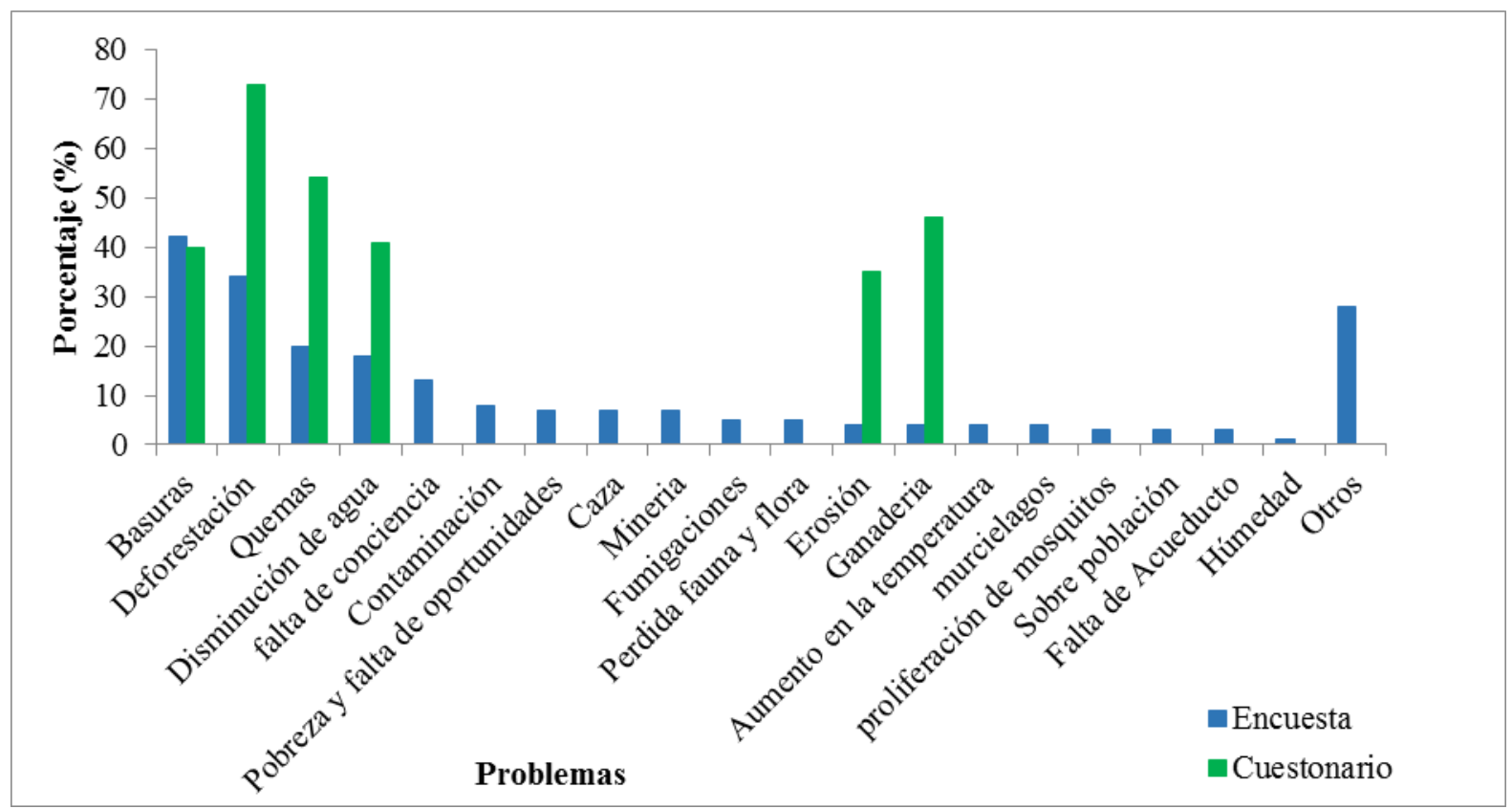

Figura 4. Problemas identificados por las personas en la Actividad "Expedición ambiental: conociendo nuestro entorno".

Espacios de interacción. Las actividades de cine y conversatorio ambiental, foro ambiental y festival del tití no sólo contribuyeron a la integración de las comunidades con distintos actores tales como la academia, las empresas, el gobierno, sino también que permitieron compartir saberes y conocer otras realidades. En las actividades participaron entidades como ISAGEN, CORPOCALDAS, Universidad Católica de Oriente (UCO), Parque Nacional Natural Selva de Florencia, Zoológico de Santa Fe y Alcaldía de Norcasia. Según el IDEA (2014) la apertura hacia la participación de los individuos y colectividades hace posible la socialización de la información en diferentes escenarios y contextos. De esta manera la información acerca del problema a solucionar, sus antecedentes y sus causas 
Bio - grafia. Escritos sobre la Biología y su Enseñanza. ISSN 2027

Edición Extraordinaria. p.p. 636 - 650

Memorias del VIII Encuentro Nacional de Experiencias en Enseñanza de la Biología y la Educación Ambiental. III Congreso Nacional de Investigación en Enseñanza de la Biología.

fluyen por canales de doble vía desde y hacia las comunidades, permitiendo un conocimiento más amplio de los diferentes aspectos del problema.

\section{CONCLUSIONES}

Las principales problemáticas ambientales en la zona son la caza, las quemas, el tráfico de especies y la ganadería, siendo esta última la que mayor impacto genera sobre la biodiversidad, evidenciado en los testimonios de las comunidades y en las grandes áreas de potreros que se pueden observar en la zona.

Las comunidades de la zona tienen un buen conocimiento sobre la fauna silvestre, no obstante, las actividades realizadas permitieron que éstos conocieran más sobre sus servicios ambientales, lo que ha llevado a una mayor valoración y protección de la misma.

La articulación con distintas entidades que trabajan en la zona entorno a la conservación de la fauna, y la interacción de éstas con las comunidades mediante el diálogo de saberes fue positiva y permitirá que se dé continuidad y sostenibilidad de los procesos ambientales que se están ejecutando.

Se contribuyó en la consolidación de líderes ambientales que transmitan los conocimientos aprendidos a otras personas de la vereda. Esto permitirá un proceso continuo y sostenible en el tiempo, generando así una actitud amigable hacia las especies amenazadas en sus comunidades.

La deforestación es una de la problemáticas más evidentes en la zona con un $73 \%$, seguido de las quemas $54 \%$ y la ganadería $46 \%$. 
Bio - grafia. Escritos sobre la Biología y su Enseñanza. ISSN 2027

Edición Extraordinaria. p.p. 636 - 650

Memorias del VIII Encuentro Nacional de Experiencias en Enseñanza de la Biología y la Educación Ambiental. III Congreso Nacional de Investigación en Enseñanza de la Biología.

El $76 \%$ de los encuestados identificó la sombra que prestan los árboles como uno de los servicios ambientales más importantes.

Un $57 \%$ de las especies identificadas por las personas de las veredas se encuentran en algún grado de amenaza.

\section{BIBLIOGRAFÍA}

- Alzate, J. (2001) Samaná en la historia. Gobernación del Departamento de Caldas: Editorial Sic. 1-5.

- Berrenechea, A. (2014). El arte de relacionarse. Recuperado de: http://www.ssreyes.org/acces/recursos/doc/Sansenet/916845114_3132009161215 .pdf. 1-31.

- Cándelo, C. R., Ortiz, R. A., Unger, B. (2003). Hacer talleres una guía práctica para capacitadores. WWF. Colombia. Recuperado de: https://www.google.com.co/url?sa=t\&rct=j\&q=\&esrc=s\&source=web\&cd=1\&cad=rja\&u act=8\&ved=0CBOQFjAA\&url=http $\% 3 A \% 2 F \% 2 F$ assets.panda.org\%2Fdownloads $\% 2 F$ in thefield_1_es.pdf\&ei=W5C2VPGwEYGDgwTanoPgDQ\&usg=AFQjCNHXmDAyNAXos EsfdFaPdLEXGz5zxA\&sig2=PaBNW01POYmzPDhnCiT01A\&bvm=bv.83640239,d.eXY. 37-38.

- Castelltort, A. V., Colaço, A., Geli, A. M., Castells, J., Pineda, B. G., Castellano, C. G.,... Gutiérrez, X. Roger. (2005). Nuevas tendencias en educación ambiental. Doctorado interuniversitario en educación ambiental.: Recuperado de: http://www.oei.es/decada/portadas/nuevas_tendencias.pdf. 
Bio - grafia. Escritos sobre la Biología y su Enseñanza. ISSN 2027

Edición Extraordinaria. p.p. 636 - 650

Memorias del VIII Encuentro Nacional de Experiencias en Enseñanza de la Biología y la Educación Ambiental. III Congreso Nacional de Investigación en Enseñanza de la Biología.

- Fundación IPADE. (2010). Pérdida de biodiversidad y pobreza. Recuperado de: http://www.fundacionipade.org/upload/pdf/Biodiversidad_pobreza.pdf.

- García, E. J. (2003). Los problemas de la educación ambiental: ¿Es posible una educación ambiental integradora? Investigación en la Escuela: 2-18. Recuperado de: http://www.ambiente.gov.ar/infotecaea/descargas/garciae01.pdf.

- Holdridge, L. R. (1982). Ecología Basada en Zonas de Vida. 1a. ed. San José, Costa Rica: IICA.

- IDEA. (2014). Herramientas metodológicas para el trabajo en educación ambiental. Recuperado el 7 de diciembre de 2014 de: http://www.virtual.unal.edu.co/cursos/IDEA/2007225/lecciones/capitulo2/01herramientasmetodologicas.htm.

- ISAGEN. (2012). Monitoreo de fauna vertebrada silvestre en zonas de influencia de los centros productivos y proyectos de ISAGEN. Informe presentado por la Universidad Católica de Oriente (Rionegro, Antioquia): 5-9.

- ISAGEN. (2014). Proyecto Manso. Informe. Recuperado de http://www.isagen.com.co/comunicados/Plegable_Manso.pdf.

- López, A: M. (2000). Las salidas de campo mucho más que una excursión. Recuperado de: http://www.educarm.es/documents/246424/461842/22_salidasdecampo.pdf/515ab 5bb-876a-4541-b5de-b5f23b103e1a. 1-4.

- Martínez, J. A. (2005). La investigación en educación ambiental como herramienta pedagógica. Revista Biocenosis Vol. 3. № 2: 1-6.

- Rojas, G., Del Águila, R., Gómez, J. \& Isola, S. (2007). La educación ambiental y la conservación de los recursos naturales en la reserva nacional Pacaya Samiria (Loreto - Perú) - Cuaderno de lectura. The Nature Conservancy, fundación peruana para la conservación de la naturaleza, Pronaturaleza (3). 39- 43.

- Terrón, A. E. (2000). La Educación ambiental ante los desafíos del siglo XXI. UPN, México, D, F.: 5- 13. 
Bio - grafia. Escritos sobre la Biología y su Enseñanza. ISSN 2027

Edición Extraordinaria. p.p. 636 - 650

Memorias del VIII Encuentro Nacional de Experiencias en Enseñanza de la Biología y la Educación Ambiental. III Congreso Nacional de Investigación en Enseñanza de la Biología.

- Tilburry, D. (1995). Environmental Education for sustainability: defining the new focus of environmental education in the 1990's, Environmental Education. Vol. 1, No. 2. 195.

- Tobasura, I. (2004). Los Medios Didácticos en la Educación Ambiental. Universidad de Caldas Revista Luna Azul 3-6.

- UCO, (2013a). Programa de educación ambiental y comunitaria dirigido a las comunidades de influencia directa de la Central Hidroeléctrica Miel I con trasvase en los ríos Guarinó y Manso. Informe presentado a ISAGEN: 4-6. 